\title{
EXTREMAL PROBLEMS IN THE CLASS OF STARLIKE FUNCTIONS ${ }^{1}$
}

\section{J. A. HUMMEL}

In an earlier paper [1] the writer showed the development of a variational method for starlike functions and applied this method to a general class of extremal problems connected with the coefficients of the function. It is the purpose of this paper to study the classes of extremal problems to which this variational method can be applied and to make some remarks as to its utility.

The class $S^{*}$ of starlike functions is the class of all functions analytic and schlicht in the unit circle, normalized by $f(0)=0$ and $f^{\prime}(0)=1$, and mapping the unit circle onto a domain which is starshaped with respect to the origin.

In [1] it was shown that given any function $f(z) \in S^{*}$ a variation can be made within the class $S^{*}$ giving a change in the function

$$
\begin{aligned}
\delta f(z)= & \epsilon\left(1-\left|z_{0}\right|^{2}\right)\left\{e^{i \theta} \frac{z f(z)}{z_{0}\left(z-z_{0}\right)}+e^{-i \theta} \frac{z f(z)}{1-z_{0}^{*} z}\right. \\
& -e^{i \theta}\left(\frac{f\left(z_{0}\right)}{z_{0} f^{\prime}\left(z_{0}\right)}\right)\left[\frac{z f^{\prime}(z)}{z-z_{0}}+\frac{f(z)}{z_{0}}\right] \\
& \left.+e^{-i \theta}\left(\frac{f\left(z_{0}\right)}{z_{0} f^{\prime}\left(z_{0}\right)}\right)^{*} \frac{z^{2} f^{\prime}(z)}{1-z_{0}^{*} z}\right\}+o(\epsilon) .
\end{aligned}
$$

where $\epsilon$ is a positive parameter tending toward zero, $\theta$ is an arbitrary real number, and $z_{0}$ is an arbitrary point in the unit circle. In this formula, and henceforth in this paper, the asterisk attached to a complex number signifies the complex conjugate of that number.

Using the identities

$$
\begin{gathered}
\frac{z}{z-z_{0}}=\frac{1}{2}\left[\frac{z+z_{0}}{z-z_{0}}+1\right], \quad \frac{z z_{0}^{*}}{1-z z_{0}^{*}}=\frac{1}{2}\left[\frac{1+z z_{0}^{*}}{1-z z_{0}^{*}}-1\right], \\
\frac{z_{0}}{z-z_{0}}=\frac{1}{2}\left[\frac{z+z_{0}}{z-z_{0}}-1\right]
\end{gathered}
$$

(1) is transformed into the more useful form

Presented to the Society, January 22, 1959; received by the editors September 25, 1959 and, in revised form, January 11, 1960.

1 This work was supported by the Office of Ordnance Research, U. S. Army, under contract DA-36-034-ORD-1486 with the University of Maryland. 
(2)

$$
\begin{aligned}
\delta f(z)= & \epsilon\left(1-\left|z_{0}\right|^{2}\right)\left\{\frac { e ^ { i \theta } } { z _ { 0 } } \left[\frac{f(z)}{2} \frac{\left(z+z_{0}\right)}{\left(z-z_{0}\right)}+\frac{f(z)}{2}\right.\right. \\
& \left.-\left(\frac{f\left(z_{0}\right)}{z_{0} f^{\prime}\left(z_{0}\right)}\right)\left(\frac{z f^{\prime}(z)}{2} \frac{\left(z+z_{0}\right)}{\left(z-z_{0}\right)}-\frac{z f^{\prime}(z)}{2}+f(z)\right)\right] \\
& +\frac{e^{-i \theta}}{z_{0}^{*}}\left[\frac{f(z)}{2} \frac{\left(1+z z_{0}^{*}\right)}{\left(1-z z_{0}^{*}\right)}-\frac{f(z)}{2}\right. \\
& \left.\left.+\left(\frac{f\left(z_{0}\right)}{z_{0} f^{\prime}\left(z_{0}\right)}\right)^{*}\left(\frac{z f^{\prime}(z)}{2} \frac{\left(1+z z_{0}^{*}\right)}{\left(1-z z_{0}^{*}\right)}-\frac{z f^{\prime}(z)}{2}\right)\right]\right\}+o(\epsilon) .
\end{aligned}
$$

In what follows we shall also need to make use of the Julia variation. If $f(z)$ maps $|z|<1$ onto a domain $D$ with boundary $C$, and if we map $C$ onto $C^{\mathbf{\Lambda}}$, the boundary of a new domain $D^{\mathbf{\Lambda}}$, by means of the mapping $w^{\boldsymbol{\Lambda}}=w+\epsilon \delta w$, where $\epsilon$ is a real parameter and $\delta w$ satisfies the requirement that it is everywhere normal to $C$, then $f_{1}(z)$ will map $|z|<1$ onto $D^{\wedge}$, where [3]

$$
f_{1}(z)=f(z)+\epsilon \oint_{C} z f^{\prime}(z) \frac{\zeta+z}{\zeta-z} \frac{\delta \omega d \omega}{2 \pi i \zeta^{2} f^{\prime}(\zeta)^{2}}+o(\epsilon) .
$$

In this formula, $\zeta$ and $\omega$ are related by $\omega=f(\zeta)$. To retain the proper normalization, we must divide (3) by $f_{1}^{\prime}(0)$, giving

$$
f^{\wedge}(z)=f_{1}(z)\left[1-\epsilon \oint_{C} \frac{\delta \omega d \omega}{2 \pi i \zeta^{2} f^{\prime}(\zeta)^{2}}\right]+o(\epsilon) .
$$

With this renormalization we obtain

$$
\delta f(z)=\epsilon \oint_{C}\left[z f^{\prime}(z) \frac{\zeta+z}{\zeta-z}-f(z)\right]\left[\frac{\delta \omega d \omega}{2 \pi i \zeta^{2} f^{\prime}(\zeta)^{2}}\right]+o(\epsilon) .
$$

In (4), $|\zeta|=1$, hence $\zeta^{*}=1 / \zeta$ giving the identity

$$
\frac{\zeta+z}{\zeta-z}=\frac{1}{2}\left[\frac{\zeta+z}{\zeta-z}+\frac{1+\zeta^{*} z}{1-\zeta^{*} z}\right] .
$$

Putting this into (4) gives the formula

$$
\begin{aligned}
\delta f(z)=\epsilon \oint_{c}\left[\frac{z f^{\prime}(z)}{2}\right. & \frac{\left(1+\zeta^{*} z\right)}{\left(1-\zeta^{*} z\right)} \\
& \left.-\frac{z f^{\prime}(z)}{2} \frac{(z+\zeta)}{(z-\zeta)}-f(z)\right]\left[\frac{\delta \omega d \omega}{2 \pi i \zeta^{2} f^{\prime}(\zeta)^{2}}\right]+o(\epsilon)
\end{aligned}
$$


for the Julia variation. Since $(d / d \theta) f\left(e^{i \theta}\right)=i e^{i \theta} f^{\prime}\left(e^{i \theta}\right)$, $i \zeta f^{\prime}(\zeta)$ is tangential to $C$ in the positive direction, hence $d \omega / 2 \pi i \zeta f^{\prime}(\zeta)$ is real and positive. Likewise $\zeta f^{\prime}(\zeta)$ is normal to $C$ and directed outward from $C$ and hence $\delta \omega / \zeta f^{\prime}(\zeta)$ is real and positive or negative as $\delta \omega$ is directed outward or inward.

In (5), the variation $\delta \omega$ must be suitably chosen if $f^{\Delta}(z)$ is to remain in the class $S^{*}$. This will easily be arranged in the application we wish to make.

The type of extremal problem which we wish to investigate is:

EXTREMAL PROBLEM. Find, in the class $S^{*}$ of starlike functions, the function $f(z)$ which maximizes (or minimizes) $\operatorname{Re}\{J[f(z)]\}$ where $J$ is a given complex valued functional on $S^{*}$.

Our methods are only suitable for functionals $J$ which behave in an essentially linear fashion for small variations in $f$. Thus we define

Definition 1. A complex valued functional $J$ on $S^{*}$ is called linear in the small if and only if for any $f$ and $f+g$ in $S^{*}$

$$
J[f+\epsilon g]=J[f]+\epsilon J_{1}[f, g]+o(\epsilon)
$$

where $J_{1}[f, g]$ is a complex valued functional depending on $f$ and $g$, but linear in $g$, i.e. if $g$ and $h$ are functions and $\alpha$ and $\beta$ are complex numbers,

$$
J_{1}[f, \alpha g+\beta h]=\alpha J_{1}[f, g]+\beta J_{1}[f, h] .
$$

One possible example of such a functional is one which is itself linear. However, if $T[f]$ is a given linear functional and $F(w)$ is a given analytic function, then $F[T(f)]$ is linear in the small, for $F[T(f+\epsilon g)]=F[T(f)+\epsilon T(g)]=F[T(f)]+\epsilon T(g) F^{\prime}[T(f)]+o(\epsilon)$.

Similarly, $T[F(f)]$ is linear in the small also, providing that $T(f)$ is continuous, for $T[F(f+\epsilon g)]=T\left[F(f)+\epsilon g F^{\prime}(f)+o(\epsilon)\right]=T[F(f)]$ $+\epsilon T\left[g F^{\prime}(f)\right]+o(\epsilon)$.

A glance at the variational formulas (2) and (5) shows that the following notation will be useful.

Definition 2. Given a functional $J$, linear in the small, with an associated linear functional $J_{1}$ as defined by (6), and given $f(z)$ in $S^{*}$ along with a point $z_{0}$ with $\left|z_{0}\right|<1$, define

$$
\begin{aligned}
R_{1}\left(z_{0}\right) & =J_{1}\left[f, f(z) \frac{z+z_{0}}{z-z_{0}}\right], \\
Q_{0} & =J_{1}[f, f(z)], \\
Q_{1}\left(z_{0}\right) & =J_{1}\left[f, z f^{\prime}(z) \frac{z+z_{0}}{z-z_{0}}\right],
\end{aligned}
$$




$$
Q_{2}=J_{1}\left[f, z f^{\prime}(z)\right]
$$

and

$$
\begin{aligned}
& R(\zeta)=R_{1}(\zeta)+Q_{0}-Q_{0}^{*}-R_{1}\left(1 / \zeta^{*}\right)^{*} \\
& Q(\zeta)=Q_{1}(\zeta)+2\left(Q_{0}-Q_{2}\right)+\left(Q_{2}+Q_{2}^{*}\right)+Q_{1}\left(1 / \zeta^{*}\right)^{*} .
\end{aligned}
$$

The reason for isolating the term $\left(Q_{0}-Q_{2}\right)$ in (8) is explained by

LEMma 1. If $f(z)$ in $S^{*}$ is a solution of the extremal problem $\operatorname{Re}\{J[f(z)]\}=$ maximum (minimum) where $J$ is linear in the small, then $Q_{0}-Q_{2}$ is real.

PROOF. The rotational variation $f^{\mathbf{\Lambda}}(z)=e^{i \epsilon} f\left(e^{-i \epsilon} z\right)$ preserves the class $S^{*}$. Here $f^{\boldsymbol{\Lambda}}(z)=(1+i \epsilon+o(\epsilon)) f[z-i \epsilon z+o(\epsilon)]=[1+i \epsilon+o(\epsilon)]$ - $\left[f(z)-i \epsilon z f^{\prime}(z)+o(\epsilon)\right]=f(z)+i \epsilon\left[f(z)-z f^{\prime}(z)\right]+o(\epsilon)$. Hence if we apply this variation to the function $f(z)$ we have the change in $J$

$$
\begin{aligned}
\delta J[f(z)] & =i \epsilon\left[J_{1}[f, f(z)]-J_{1}\left[f, z f^{\prime}(z)\right]\right]+o(\epsilon) \\
& =i \epsilon\left(Q_{0}-Q_{2}\right)+o(\epsilon)
\end{aligned}
$$

If the function $f(z)$ maximizes $\operatorname{Re}\{J\}$, then this variation must have a nonpositive real part for all $\epsilon$, positive or negative. But this means that $\operatorname{Re}\left\{i\left(Q_{0}-Q_{2}\right)\right\}=0$, which proves the lemma.

THEOREM 1. If $f(z)$ in $S^{*}$ is a solution of the extremal problem $\operatorname{Re}\{J[f(z)]\}=$ maximum (or minimum) where $J$ is linear in the small, then $f(z)$ satisfies the differential equation

$$
\frac{\zeta f^{\prime}(\zeta)}{f(\zeta)} R(\zeta)=Q(\zeta)
$$

for all $\zeta$ in $|\zeta|<1$.

Proof. If $f(z)$ maximizes $\operatorname{Re}\{J[f(z)]\}$, then for any variation of $f(z)$ in $S^{*}$ we must have $\operatorname{Re}\{\delta J[f(z)]\} \leqq 0$. Using the variation (2) we have

$$
\begin{aligned}
\delta J[f(z)]= & \epsilon \frac{\left(1-\left|z_{0}\right|^{2}\right)}{2}\left\{\frac { e ^ { i \theta } } { z _ { 0 } } \left[R_{1}\left(z_{0}\right)+Q_{0}\right.\right. \\
& \left.-\left(\frac{f\left(z_{0}\right)}{z_{0} f^{\prime}\left(z_{0}\right)}\right)\left(Q_{1}\left(z_{0}\right)-Q_{2}+2 Q_{0}\right)\right] \\
& \left.-\frac{e^{-i \theta}}{z_{0}^{*}}\left[R_{1}\left(1 / z_{0}^{*}\right)+Q_{0}+\left(\frac{f\left(z_{0}\right)}{z_{0} f^{\prime}\left(z_{0}\right)}\right)^{*}\left(Q_{1}\left(1 / z_{0}^{*}\right)+Q_{2}\right)\right]\right\} \\
& +o(\epsilon) .
\end{aligned}
$$


Since the real part of any quantity is the same as the real part of its complex conjugate, this gives

$$
\begin{aligned}
\operatorname{Re}\{\delta J[f(z)]\}= & \epsilon \frac{\left(1-\left|z_{0}\right|^{2}\right)}{2} \operatorname{Re}\left\{\frac{e^{i \theta}}{z_{0}}\left[R\left(z_{0}\right)-\left(\frac{f\left(z_{0}\right)}{z_{0} f^{\prime}\left(z_{0}\right)}\right) Q\left(z_{0}\right)\right]\right\} \\
& +o(\epsilon) .
\end{aligned}
$$

The right hand side of this equation must be nonpositive for all $\theta$, hence the expression in square brackets must be zero for the extremal function $f(z)$ and for any $z_{0}$ in the unit circle. But this is equivalent to (9), the desired conclusion of the theorem.

By making further restrictions on the form of $R(z)$ and $Q(z)$ we can obtain the following stronger results.

THEOREM 2. If $R(\zeta)$ and $Q(\zeta)$ are finite, nonzero and continuous at a point $\zeta=\zeta_{0}$ on the unit circle, then the extremal function $f(\zeta)$ maps a segment of the arc of the unit circle containing the point $\zeta=\zeta_{0}$ onto a radial line segment.

Proof. With the given hypotheses, there must be a segment of arc $\gamma$ of the unit circle, containing $\zeta_{0}$ such that $R(\zeta)$ and $Q(\zeta)$ are defined, finite, and nonzero on $\gamma$. But if $\zeta$ is any point of $\gamma$, then $\zeta=1 / \zeta^{*}$, and from (8) we see that $R(\zeta)$ must be pure imaginary. Likewise, $Q(\zeta)$ must be purely real on each point of $\gamma$ (from Lemma $1, Q_{0}-Q_{2}$ is purely real). Thus on each point of $\gamma, \zeta f^{\prime}(\zeta) / f(\zeta)$ $=Q(\zeta) / R(\zeta)$ is defined, continuous, and purely imaginary. Since $\zeta f^{\prime}(\zeta) / f(\zeta)$ is analytic inside the unit circle and continuous on $\gamma$, the conclusion of the theorem follows from the observation that

$$
\frac{\partial \arg f\left(r e^{i \theta}\right)}{\partial \theta}=\operatorname{Re}\left\{\frac{r e^{i \theta} f^{\prime}\left(r e^{i \theta}\right)}{f\left(r e^{i \theta}\right)}\right\} .
$$

THEOREM 3. Let the extremal problem $\operatorname{Re}\{J[f(z)]\}=$ maximum (or minimum) be such that $J$ is linear in the small, and such that $R_{1}(\zeta)$ and $Q_{1}(\zeta)$ defined in (7) are both rational functions of the same order $n$, both being regular on $|\zeta|=1$. Further, let $R(\zeta)$ defined in (8) have $2 p$ zeros which are not on $|\zeta|=1$. Then $f(z)$, the extremal function, maps $|z|<1$ onto the w-plane cut by $n-p$ or fewer radial slits. Indeed

$$
f(z)=z / \prod_{\nu=1}^{m}\left(1-\kappa_{\nu} z\right)^{\mu_{\nu}}
$$

where $m \leqq n-p$, each $\mu_{\nu}$ is real and positive with $\sum \mu_{\nu}=2$, and for each $\nu,\left|\kappa_{\nu}\right|=1$. 
Proof. We first remark that the hypotheses of this theorem are satisfied by the extremal problems which are normally considered.

If $R_{1}(\zeta)$ is rational, then so is $R_{1}\left(1 / \zeta^{*}\right)^{*}$. As a result, $R(\zeta)$ is rational and of order at most $2 n$. Clearly, $R(\zeta)$ is regular on $|\zeta|=1$. Since $R(\zeta)=-R\left(1 / \zeta^{*}\right)^{*}$, any zeros of $R(\zeta)$ not on $|\zeta|=1$ must occur in pairs, conjugate with respect to the unit circle.

In a similar fashion we see that $Q(\zeta)$ is rational, of order at most $2 n$, and is regular on $|\zeta|=1$.

Now $z f^{\prime}(z) / f(z)=Q(z) / R(z)$ is regular and nonzero for $|z|<1$. But also, except for at most a finite number of poles, $Q(z) / R(z)$ is purely imaginary on $|z|=1$. Hence by the Schwarz reflection principle, $z f^{\prime}(z) / f(z)$ is regular and nonzero outside of the unit circle also. Thus all zeros and poles must be on $|z|=1$ and hence all poles and zeros, not on the unit circle, of $R(z)$ and $Q(z)$ must cancel. We conclude that $Q(z) / R(z)$ is a rational function of order at most $2(n-p)$, with all of its zeros and poles on $|z|=1$, all such zeros arising from zeros of $Q(z)$.

From Theorem $2, f(z)$ must map the boundary of the unit circle onto a set of radial slits. In particular, the image of the unit circle can have no exterior. The tip of each slit corresponds to a zero of $Q(z)$ on $|z|=1$ and the point at infinity (between two adjacent slits) corresponds to a simple pole of $Q(z) / R(z)$. Thus there are at most $2(n-p)$ slits.

Expanding $z f^{\prime}(z) / f(z)$ according to its poles gives

$$
\frac{z f^{\prime}(z)}{f(z)}=1+\sum_{\nu=1}^{m} \frac{\mu_{\nu} \kappa_{\nu} z}{1-\kappa_{\nu} z}, \quad m \leqq 2(n-p),
$$

where each of the $m$ slits gives rise to one term in the sum. Each $\left|\kappa_{\nu}\right|=1$, each $\mu_{\nu}$ is real and positive and $\sum \mu_{\nu}=2$ (since $\pi \mu_{\nu}$ is the angle between two adjacent slits and the sum of all these angles is $2 \pi)$. The summation in (11) accounts for all poles, hence the remaining term is a constant, which must be 1 since $z f^{\prime}(z) / f(z)=1$ at $z=0$.

Dividing (11) by $z$ and integrating gives

$$
f(z)=z / \prod_{\nu=1}^{m}\left(1-\kappa_{\nu} z\right)^{\mu_{\nu}}
$$

as asserted in (10).

It remains only to show that $m \leqq(n-p)$. This is done by means of the Julia Variation (5). From (5) and (7) we have

$$
\delta J[f(z)]=-\frac{\epsilon}{2} \oint_{c}\left[Q_{1}\left(1 / \zeta^{*}\right)+Q_{1}(\zeta)+2 Q_{0}\right]\left[\frac{\delta \omega d \omega}{2 \pi i \zeta^{2} f^{\prime}(\zeta)^{2}}\right]+o(\epsilon) .
$$


As explained above, the factor $\phi d \omega=\left[\delta \omega d \omega / 2 \pi i \zeta^{2} f^{\prime}(\zeta)^{2}\right]$ is pure real, hence

$$
\begin{aligned}
\delta & \operatorname{Re}\{J[f(z)]\} \\
& =-\frac{\epsilon}{2} \oint_{c} \operatorname{Re}\left\{Q_{1}\left(1 / \zeta^{*}\right)+Q_{1}(\zeta)+2\left(Q_{0}-Q_{2}\right)+Q_{2}+Q_{2}\right\} \phi d \omega+o(\epsilon) \\
& =-\frac{\epsilon}{2} \oint_{c} \operatorname{Re}\left\{Q_{1}\left(1 / \zeta^{*}\right)^{*}+Q_{1}(\zeta)+2\left(Q_{0}-Q_{2}\right)+Q_{2}+Q_{2}^{*}\right\} \phi d \omega+o(\epsilon) \\
& =-\frac{\epsilon}{2} \oint_{C} \operatorname{Re}\{Q(\zeta)\} \phi d \omega+o(\epsilon),
\end{aligned}
$$

or finally

$$
\delta \operatorname{Re}\{J[f(z)]\}=-\frac{\epsilon}{2} \oint_{c} Q(\zeta)\left[\frac{\delta \omega d \omega}{2 \pi i \zeta^{2} f^{\prime}(\zeta)^{2}}\right]+o(\epsilon) .
$$

Here we made use of the fact that the real part of any quantity is the same as the real part of its complex conjugate, and also that $Q(\zeta)$ is purely real on $|\zeta|=1$.

The variation to be used in (12) consists of "hinging" one of the radial slits at some point $\omega_{0}$ and expanding it out to a wedge from that point out to $\infty$. The displacement, $\epsilon \delta \omega$, in this case is $\pm i \epsilon\left(\omega-\omega_{0}\right)$ along the portion of one side of the slit from $\omega_{0}$ to $\infty$, and zero everywhere else, the sign being chosen so that $\delta \omega$ is directed inward. The parameter $\epsilon$ must be chosen small enough that the angle of the wedge so formed is less that the angle between the two adjacent rays.

With this variation, $\left[\delta \omega d \omega / 2 \pi i \zeta^{2} f^{\prime}(\zeta)^{2}\right]$ is real and positive. Further, since $d \omega=f^{\prime}(\zeta) d \zeta$, and $\delta \omega=i\left[f(\zeta)-f\left(\zeta_{0}\right)\right]$ this expression behaves like $\left[\zeta \cdot \zeta f^{\prime}(\zeta) / f(\zeta)\right]^{-1} d \zeta$ as $f(\zeta)$ tends to $\infty$. But $\zeta f^{\prime}(\zeta) / f(\zeta)$ has a simple pole at this point and hence $\left[\delta \omega d \omega / 2 \pi i \zeta^{2} f^{\prime}(\zeta)^{2}\right]$ tends to zero as we go out toward $\infty$. Now if $\omega_{0}$ is far enough out along the slit so that $Q(\zeta)$ is of constant sign along the varied portion, then from (12), since $\delta \operatorname{Re}\{J[f(z)]\} \leqq 0$ for the extremal function, $Q(\zeta)$ must be positive for $\zeta$ corresponding to points along the varied portion. Thus $Q(\zeta)$ is positive in a deleted neighborhood of each $\zeta$ which maps to $\infty$. But this means it must have an even number of zeros between each such pair of points. Since there is one zero for the tip of the slit, each slit must give rise to at least two zeros of $Q(\zeta)$. There are at most $2(n-p)$ zeros in all, hence there can be at most $n-p$ slits. This completes the proof of the theorem.

A few examples may help clarify the use of the variational method. An application to coefficient problems may be found in [2]. Another 
application is to the already solved problem: what is the set of all possible values of $f\left(z_{0}\right)$ for a fixed $z_{0}$ and for all $f \in S^{*}$ ? The problem is somewhat simplified if we ask instead for the set of all values of $\log f\left(z_{0}\right)$. For if $\log f\left(z_{0}\right)=A$ and $\log g\left(z_{0}\right)=B$, then the function $h(z)$ $=[f(z)]^{1-\alpha}[g(z)]^{\alpha}$ has the property that $\log h\left(z_{0}\right)=(1-\alpha) A+\alpha B$, while if $0 \leqq a \leqq 1, f$ and $g \in S^{*}$ implies $h \in S^{*}$ since $z h^{\prime} / h=(1-\alpha) z f^{\prime} / f$ $+\alpha z g^{\prime} / g$. Thus the set of all possible values of $\log f\left(z_{0}\right)$ is convex. To find a point on the boundary of this region, we merely choose a direction, defined by a fixed complex $\lambda$ and find the $f(z) \in S^{*}$ which maximizes $\operatorname{Re}\left\{\lambda \log f\left(z_{0}\right)\right\}$. Here, $J[f(z)]=\left\{\lambda \log f\left(z_{0}\right)\right\}$ and

$$
\begin{aligned}
J[f(z)+\epsilon g(z)] & =\lambda \log \left[f\left(z_{0}\right)+\epsilon g\left(z_{0}\right)\right] \\
& =\lambda \log f\left(z_{0}\right)+\epsilon \lambda g\left(z_{0}\right) / f\left(z_{0}\right)+o(\epsilon) .
\end{aligned}
$$

Hence $J_{1}[f, g]=\lambda g\left(z_{0}\right) / f\left(z_{0}\right)$ and thus

$$
\begin{aligned}
R_{1}(\zeta) & =\lambda \frac{z_{0}+\zeta}{z_{0}-\zeta}, & Q_{1}(\zeta) & =\lambda \frac{z_{0} f^{\prime}\left(z_{0}\right)}{f\left(z_{0}\right)} \frac{z_{0}+\zeta}{z_{0}-\zeta}, \\
Q_{0} & =\lambda, & Q_{2} & =\lambda \frac{z_{0} f^{\prime}\left(z_{0}\right)}{f\left(z_{0}\right)} .
\end{aligned}
$$

Here $R_{1}(\zeta)$ and $Q_{1}(\zeta)$ satisfy the hypotheses of Theorem 3 with $n=1$. Therefore the extremal function has but one slit, and is indeed the Koebe function. The boundary of the region in question is thus given by

$$
\log f\left(z_{0}\right)=\log z_{0}-2 \log (1-\kappa z)
$$

as $\kappa$ takes on all values on the unit circle, $|\kappa|=1$.

As a second example, let us look for the set of all possible values of $f\left(z_{1}\right) / f\left(z_{2}\right)$ for a fixed pair of points $z_{1}$ and $z_{2}$ and for all possible $f \in S^{*}$. Again the set of all possible values of $\log \left[f\left(z_{1}\right) / f\left(z_{2}\right)\right]$ can be shown to be convex, exactly as above. For the extremal problem $\operatorname{Re}\left\{\lambda \log \left[f\left(z_{1}\right) / f\left(z_{2}\right)\right]\right\}=$ maximum, we have

$$
J_{1}[f, g]=\lambda\left[\frac{g\left(z_{1}\right)}{f\left(z_{1}\right)}-\frac{g\left(z_{2}\right)}{f\left(z_{2}\right)}\right]
$$

and hence

$$
R_{1}(\zeta)=\lambda\left[\frac{z_{1}+\zeta}{z_{1}-\zeta}-\frac{z_{2}+\zeta}{z_{2}-\zeta}\right], Q_{0}=0 .
$$

Here $R_{1}(0)=R_{1}(\infty)=0$, hence $R(\zeta)$ has two of its four zeros at 0 and $\infty$. Thus from Theorem 3, the extremal function has but one 
slit and the set of possible values of $f\left(z_{1}\right) / f\left(z_{2}\right)$ is the closed region bounded by the curve

$$
\frac{f\left(z_{1}\right)}{f\left(z_{2}\right)}=\frac{z_{1}}{z_{2}}\left[\frac{1-\kappa z_{2}}{1-\kappa z_{1}}\right]^{2}, \quad|\kappa|=1 .
$$

In closing we note that Theorem 3 can be used to determine the type of problem which can be attacked effectively by means of the variational method. For a given extremal problem, it is usually a simple matter to determine the functional $J_{1}[f, g]$ and from this to evaluate $R_{1}(\zeta)$ and $Q_{1}(\zeta)$. Theorem 3 then immediately gives the form of the extremal function.

Any difficulty will usually arise in one of two ways. First, Theorem 3 gives only an upper bound for the number of slits. The accuracy of this number is guaranteed only when it equals one, unless we can tell how many zeros $R(\zeta)$ has in the interior of the unit circle. Frequently this cannot be done since the coefficients of terms in $R(\zeta)$ depend on unknown quantities determined by the extremal function itself.

A second difficulty, is that even if we know the exact form of the extremal function, this does not always answer questions we may have about the actual value of the functional for this extremal function, which is often the item of prime interest.

For example, the problem of Marx [4] is similar to the two examples given above. What is asked for is the set of all possible values of $\log f^{\prime}\left(z_{0}\right)$ for a fixed $z_{0}$ and for all $f \in S^{*}$. It can easily be shown with the help of Theorem 3 that the extremal functions in this case have at most two slits. (This result was obtained much earlier by Robinson [5].) But the conjecture of Marx is that the extremal functions have only one slit, and this result, although already partially proved [5], does not seem to be obtainable by elementary means from the variational method.

\section{REFERENCES}

1. J. A. Hummel, $A$ variational method for starlike functions, Proc. Amer. Math. Soc. vol. 9 (1958) pp. 82-87.

2. - The coefficient regions of starlike functions, Pacific J. Math. vol. 7 (1957) pp. 1381-1389.

3. G. Julia, Sur une equation aux dérivées fonctionnelles liée à la représentation conforme, Ann. Sci. Ecole Norm. Sup. (3) vol. 39 (1922) pp. 1-28.

4. A. Marx, Untersuchungen über schlichte Abbildungen, Math. Ann. vol. 107 (1932) pp. 40-67.

5. R. M. Robinson, Extremal problems for star mappings, Proc. Amer. Math. Soc. vol. 6 (1955) pp. 364-377.

UNIVERSITY OF MARYLAND 